\title{
Dental and Periodontal Treatment Need after Dental Clearance Is Not Associated with the Outcome of Induction Therapy in Patients with Acute Leukemia: Results of a Retrospective Pilot Study
}

\author{
Gerhard Schmalz, ${ }^{1}$ Lulzim Tulani, ${ }^{1}$ Rilana Busjan, ${ }^{1}$ Rainer Haak, ${ }^{1}$ Tanja Kottmann, \\ Lorenz Trümper, ${ }^{3}$ Justin Hasenkamp, ${ }^{3}$ and Dirk Ziebolz $\mathbb{(}^{1}$ \\ ${ }^{1}$ Dept. of Cariology, Endodontology and Periodontology, University of Leipzig, Leipzig, Germany \\ ${ }^{2}$ Clinical Research Organization, Hamm, Germany \\ ${ }^{3}$ Clinic for Hematology and Medical Oncology, University Medical Center Goettingen, Göttingen, Germany \\ Correspondence should be addressed to Dirk Ziebolz; dirk.ziebolz@medizin.uni-leipzig.de
}

Received 27 February 2020; Accepted 2 April 2020; Published 21 April 2020

Academic Editor: Elvira Grandone

Copyright (@) 2020 Gerhard Schmalz et al. This is an open access article distributed under the Creative Commons Attribution License, which permits unrestricted use, distribution, and reproduction in any medium, provided the original work is properly cited.

\begin{abstract}
This retrospective pilot study aimed to detect whether remaining dental/periodontal treatment need and periodontal inflammation after dental clearance would be associated with the initial therapy outcome of adult patients with acute leukemia undergoing induction chemotherapy. Different parameters were assessed from the patients' records: initial blood parameters, blood parameters during initial chemotherapy, leukemia/therapy related complaints, duration of fever, microbiological findings (blood and urine), as well as patients' survival. Dental treatment need was defined as the presence of at least one carious tooth; periodontal treatment need was determined by the presence of probing depth $\geq 3.5 \mathrm{~mm}$ in at least two sextants. To reflect periodontal inflammation, the periodontal inflamed surface area (PISA) was applied. Thirty-nine patients were included. A dental treatment need of $75 \%$ and periodontal treatment need of $76 \%$ as well as an average PISA of $153.18 \pm 158.09$ were found. Only two associations were detected: periodontal treatment need was associated with thrombocyte count after 7 days $(p=0.03)$, and PISA was associated with erythrocyte count three days after induction of therapy $(p=0.01)$. It can be concluded that remaining dental and periodontal treatment need as well as periodontal inflammation after dental clearance is not associated with the outcome of induction therapy in adult patients with acute leukemia.
\end{abstract}

\section{Introduction}

Acute leukemia is a highly progressive and severe disease which takes a fatal course if no appropriate treatment is applied [1]. Thereby, induction chemotherapy is still the standard therapy for the majority of affected patients [2]. Although this therapeutic intervention is often the only chance to save patients' life, infectious complications caused by the immunosuppressive effect of chemotherapy are a major problem, leading to increased mortality [3]. As one potential entry point of bacteria in the blood, the oral cavity and related infectious diseases are of relevance as an important source of bacteraemia
[4]. In consequence, the dental clearance prior to chemotherapy is a regular recommendation $[5,6]$. This is supported by the fact that especially invasive oral treatment measures of patients undergoing chemotherapy can cause serious consequences [7]. Several considerations must be made in this context. The underlying leukemic disease can often lead to the affection of haematopoiesis, resulting in pancytopenia (anaemia, granulocytopenia, and thrombocytopenia) and thus to increased susceptibility to infections and increased bleeding tendency $[1,8,9]$. Furthermore, a delay in anticancer therapy caused by dental rehabilitation should be avoided [6]. These factors are important limitations of possible dental surgical 
interventions before the onset of induction chemotherapy. Thus, dental clearance is often primarily restricted to the elimination of dental foci with an increased risk of causing systemic complications $[5,10]$.

An available decision analysis model showed that additional death of $0.18 \%$ of patients can be avoided by dental treatment prior to chemotherapy, and a reduction in systemic infections by approximately one-third might be achievable $[5,10]$. However, the clinical data supporting these findings are rare, especially regarding the effect of remaining treatment need after dental clearance. Recent examinations showed that, on the one hand, oral diseases might show a limited increase in the risk of bacteraemia in these patients [11]. On the other hand, it has been found that poor oral health had no influence on the outcome of induction therapy [12]. Accordingly, the real clinical impact of oral health situation on infectious complications and therapy outcome seems not clarified. In this context, increased risk during dental procedures in patients before chemotherapy [6] and a potential reduction of patients' quality of life by radical dental rehabilitation (e.g., numerous tooth extractions) [13] must be considered in the dental care of these patients.

The current pilot study was performed to detect whether a remaining treatment need (dental and periodontal) as well as periodontal inflamed surface area (PISA) after a previously performed dental clearance would affect the outcome of induction chemotherapy in adult patients with acute leukemia. It was hypothesized that remaining treatment need and periodontal inflammation after dental clearance would not affect therapy outcome and mortality.

\section{Materials and Methods}

2.1. Study design. The current examination was performed as a retrospective, observational pilot study and has been reviewed and approved by the Ethics Committee of the University Medical Center Goettingen, Germany (No. 30/1/ 14). All Patients provided their written informed consent for participation in the current study. All participants were already part of a cross-sectional evaluation of oral health and microbiological parameters in adult patients suffering from newly diagnosed acute leukemia. Accordingly, general oral health parameters and microbiological findings have already been published before [14].

2.2. Patients. Between January 2015 and April 2015, hospitalized patients suffering from acute leukemia in the Clinic of Hematology and Oncology of the University Medical Center Goettingen to receive induction chemotherapy were recruited. The assessment of clinical oral parameters was performed by one experienced dentist in course of the previous cross-sectional study [14]. Out of these patients, the participants for this retrospective analysis were selected using specific inclusion and exclusion criteria. Accordingly, a mandatory condition for participation in the current study was a history of induction chemotherapy because of acute leukemia in the Clinic of Hematology and Oncology of the University Medical Center Goettingen. Before the onset of chemotherapy, a dental clearance of potential infectious dental foci was performed by the dentist of included patients. Thereby, dental conditions that pose a risk for immediate acutization or systemic spreading should be eliminated prior to onset of induction therapy. The oral examination for this current study was performed after the patient's appointment for dental clearance. The exclusion criteria were equal as in the previous cross-sectional examination: age below 18 years, a poor general health condition that prohibited oral examination, pregnancy, and autoimmune diseases such as rheumatoid arthritis or chronic bowel diseases.

2.3. Acquisition of medical and leukemia related data. The patients' records of all participants were screened, and the following parameters were extracted for analysis:

(i) Initial blood parameters before therapy onset: C-reactive protein (CRP), lactate dehydrogenase (LDH), activated partial thromboplastin time (aPTT), hemoglobin, hematocrit, and count of erythrocytes, leukocytes, and thrombocytes

(ii) Course of blood parameters during initial therapy: count of erythrocytes, leukocytes, and thrombocytes at three and seven days after therapy onset

(iii) Duration of thrombopenia, erythropenia, leukopenia, neutropenia, and fever during initial therapy

(iv) The highest CRP value during initial therapy period (CRP max)

(v) The detection of positive microbiological findings in the culture of blood and urine

(vi) leukemia/therapy related complaints including weakness, tachycardia, vertigo/nausea, and dyspnea

(vii) death of participants during therapy and the reason why participants died

2.4. Oral Examination. The course of oral examination has been performed once under standardized conditions, as described previously [14]. In brief, dental health assessment included decayed (D), missing (M), and filled (F) teeth index (DMF-T) according to WHO [15]. Based on the presence of carious teeth showing cavitation of the tooth surface, which required dental intervention, dental treatment need was defined. Furthermore, the periodontal status included the periodontal probing depths (PPD), the occurrence of bleeding (Bleeding on probing $=\mathrm{BOP}$ ), as well as the clinical attachment loss (CAL). According to the periodontal screening index (PSI), the presence of PPD $\geq 3.5 \mathrm{~mm}$ in at least two sextants was interpreted as a periodontal treatment need [16]. Furthermore, the periodontal inflamed surface area (PISA), which enables quantification of inflammatory periodontal burden, was assessed [17].

2.5. Statistical Analysis. Statistical analysis was performed using SPSS for Windows, version 24.0 (SPSS Inc., USA). The samples were tested for normal distribution with the Shapiro-Wilk test. In a comparison of two independent, 
normally distributed samples, a $t$-test was used, while nonnormally distributed samples were analyzed with Mann-Whitney $U$-test. Categorized data were analyzed using Fisher's exact tests or chi-square tests, respectively. The significance level was set at $\alpha=5 \%$.

\section{Results and Discussion}

3.1. Patients, Treatment Need, and PISA. A total of 39 patients suffering from acute leukemia with a mean age of $55.61 \pm 17.01$ years were included in the current study. A dental treatment need was found for $75 \%$ of study participants, while $76 \%$ had a need for periodontal treatment. An average PISA of $153.18 \pm 158.09$ was determined for the patients (Table 1).

\subsection{Associations between Treatment Need/PISA and Leukemia} Parameters. For dental treatment need, no associations to the initial blood values and the initial therapy outcome parameters were found. Furthermore, the positive microbiological culture in blood and urine was not related to the presence of dental treatment need ( $p>0.05$; Table 2$)$. Patients with periodontal treatment need showed a statistically significant lower thrombocyte count at seven days after therapy onset $(43.0 \pm 44.5$ vs. $131.3 \pm 108.5, p=0.03)$. Further associations between periodontal treatment need and blood parameters, therapy related factors, and microbiological findings could not be detected ( $p>0.05$; Table 2). Based on the distribution by the median of PISA, participants with a PISA $>98 \mathrm{~mm}^{2}$ had a lower erythrocyte count at three days after induction of therapy $(3.0 \pm 0.5$ vs. $3.9 \pm 1.5$, $p=0.01)$. Further associations for PISA with blood values or therapy related parameters were not found $(p>0.05$; Table 3).

3.3. Associations between Treatment Need/PISA and Complaints. For the assessed treatment related complaints, including weakness, tachycardia, vertigo/nausea, and dyspnea, no associations to either dental/periodontal treatment need or to the PISA were detected $(p>0.05$; Table 4).

3.4. Associations between Treatment Need/PISA and Mortality. From the 39 included patients receiving induction chemotherapy, 6 patients died within the observational period. Half of them $(n=3)$ died because of the cancerous disease itself, while the cause of death for two other patients was an infection. The presence of dental or periodontal treatment need as well as the PISA was not associated with the mortality of participants $(p>0.05$; Table 4$)$.

\section{Discussion}

4.1. Summary of the Main Results. Despite a previous dental clearance, dental and periodontal treatment need of the examined patients with acute leukemia was high. No substantial associations of dental/periodontal treatment need as well as PISA with initial blood parameters, outcome parameters of initial therapy, complaints of the patients, microbiological findings, and mortality were found.

4.2. Comparison with Available Literature. Due to severe immunosuppression because of the underlying disease as well as its therapy, patients with acute leukemia are at increased risk for severe infections [3]. In this respect, bacteraemia from different sources are an important concern. Oral diseases, especially periodontal inflammation, are associated with both growth of bacteria with high pathological potential and increased permeability of inflamed epithelial surface [18]. These factors enable a large amount of bacteria to enter the blood circulation, even during daily routine procedures like chewing or oral hygiene measures $[19,20]$. High prevalence of gingivitis/periodontitis was concluded to be an independent risk factor for infectious complications during chemotherapy [21]. Furthermore, dental interventions, especially oral surgery during chemotherapy, can cause serious consequences like bleeding complications [7]. Accordingly, dental rehabilitation prior to induction chemotherapy is recommended to avoid both infectious complications and the necessity of dental interventions during therapy $[5,6]$. This is also supported by a survey of dental clinicians, which prioritize dental rehabilitation before induction therapy [22].

The previous cross-sectional study of this working group found a poor oral health condition in adult patients with untreated acute leukemia and concluded improved dental care to be necessary for these patients [14]. However, it was unclear whether this oral situation would affect the therapy outcome, occurrence of infections, or mortality of the patients. Therefore, the current study retrospectively assessed these parameters. As the first aspect, there were no associations for treatment need (dental and periodontal) and PISA with initial hematological blood parameters. These findings are in line with an examination by Angst et al. [23]. In generally healthy individuals, the reflection of periodontal inflammation in blood values might be comparatively low [24]. Accordingly, this appears to be similar in the group of patients with acute leukemia in the current study. Furthermore, the initial therapy outcome, reflected by the extent and duration of pancytopenia and fever, was examined regarding its associations to the oral parameters. Although two types of significance were detected, the vast majority of examined parameters were found to be unaffected by treatment need and periodontal inflammation. Only one comparable examination is available, showing that poor oral health did not affect the induction outcome in children with acute leukemia [12]. This underlines the assumption that oral condition aside from dental infectious foci might be just a minor influential factor for hematological therapy outcomes. However, considering the literature [17], the patients within the current study were found to suffer from a comparably small PISA. Therefore, this small inflammatory burden might blur an effect on systemic factors and therapy outcomes.

A parameter of high clinical importance and relevance is the occurrence of infectious complications during induction 
TABle 1: Patients' characteristics including age, gender, and treatment need, as well as PISA.

\begin{tabular}{lcr}
\hline & & Leukemia $(n=39)$ \\
\hline Gender $(n=39)$ & $\begin{array}{c}\text { Male } n(\%) \\
\text { Female } n(\%)\end{array}$ & $\begin{array}{c}19(49) \\
20(51)\end{array}$ \\
\hline Age in years $(n=39)$ mv \pm sd (median) & & $55.61 \pm 17.01(57)$ \\
\hline \multirow{2}{*}{ Form of leukemia } & Acute myeloid leukemia (AML) & $26(67)$ \\
& Acute lymphoblastic leukemia (ALL) & $13(33)$ \\
Dental treatment need $(n=39)$ & Yes & $29(75)$ \\
Periodontal treatment need $(n=34)$ & No & $10(25)$ \\
\hline PISA $(n=34)$ mv \pm sd (median) & Yes & $25(74)$ \\
\hline
\end{tabular}

mv: mean value, sd: standard deviation, PISA: periodontal inflamed surface area.

TABLE 2: Initial blood parameters and therapeutic process depending on dental as well as periodontal treatment need.

\begin{tabular}{|c|c|c|c|c|c|c|c|}
\hline \multirow{2}{*}{ Parameter } & & \multicolumn{3}{|c|}{ Periodontal treatment need } & \multicolumn{3}{|c|}{ Dental treatment need } \\
\hline & & Yes $(n=25)$ & No $(n=9)$ & $p$ value & Yes $(n=29)$ & No $(n=10)$ & $p$ value \\
\hline \multicolumn{8}{|l|}{ Initial blood parameters } \\
\hline CRP $(\mathrm{mg} / \mathrm{l})$ & & $41.9 \pm 41.7$ & $21.1 \pm 17.6$ & 0.48 & $40.3 \pm 39.7$ & $46.8 \pm 90.7$ & 0.21 \\
\hline $\mathrm{LDH}(\mathrm{U} / \mathrm{I})$ & & $642.6 \pm 679.9$ & $795.8 \pm 1116.9$ & 0.73 & $723.2 \pm 870.4$ & $597.8 \pm 438.5$ & 0.71 \\
\hline aPTT (sec) & & $28.4 \pm 6.5$ & $28.6 \pm 2.7$ & 0.90 & $29.1 \pm 6.8$ & $28.0 \pm 3.6$ & 0.58 \\
\hline Hemoglobin (g/dl) & & $9.8 \pm 1.3$ & $10.7 \pm 2.3$ & 0.29 & $10.1 \pm 1.6$ & $10.0 \pm 1.6$ & 0.95 \\
\hline Hematocrit (\%) & & $28.6 \pm 4.3$ & $31.3 \pm 6.8$ & 0.29 & $29.6 \pm 4.9$ & $29.1 \pm 5.3$ & 0.78 \\
\hline \multicolumn{8}{|l|}{ Initial therapy parameters } \\
\hline \multirow{3}{*}{ Erythrocytes $\left(10^{3} / \mu \mathrm{l}\right)$} & Initial & $3.2 \pm 0.6$ & $3.5 \pm 0.9$ & 0.51 & $3.4 \pm 0.7$ & $3.2 \pm 0.6$ & 0.31 \\
\hline & 3 days & $3.4 \pm 1.2$ & $2.5 \pm 1.7$ & 0.85 & $3.1 \pm 0.9$ & $4.0 \pm 1.9$ & 0.14 \\
\hline & 7 days & $2.9 \pm 0.5$ & $2.4 \pm 1.6$ & 0.21 & $3.1 \pm 1.0$ & $2.9 \pm 0.7$ & 0.33 \\
\hline \multirow{3}{*}{ Thrombocytes $\left(10^{3} / \mu \mathrm{l}\right)$} & Initial & $66.4 \pm 69.1$ & $153.4 \pm 136.9$ & 0.07 & $67.7 \pm 76.0$ & $145.4 \pm 133.6$ & 0.12 \\
\hline & 3 days & $59.5 \pm 52.8$ & $147.4 \pm 135.4$ & 0.19 & $68.0 \pm 77.7$ & $118.3 \pm 110.2$ & 0.63 \\
\hline & 7 days & $43.0 \pm 44.5$ & $131.3 \pm 108.5$ & 0.03 & $53.2 \pm 67.1$ & $83.3 \pm 83.6$ & 0.50 \\
\hline \multirow{3}{*}{ Leukocytes $\left(10^{3} / \mu \mathrm{l}\right)$} & Initial & $18.4 \pm 35.7$ & $35.3 \pm 59.7$ & 0.31 & $25.2 \pm 46.3$ & $15.3 \pm 24.1$ & 0.50 \\
\hline & 3 days & $14.4 \pm 27.6$ & $31.1 \pm 65.5$ & 0.77 & $19.9 \pm 41.2$ & $19.2 \pm 41.0$ & 0.89 \\
\hline & 7 days & $6.9 \pm 15.3$ & $10.8 \pm 12.1$ & 0.07 & $8.3 \pm 15.6$ & $2.7 \pm 3.2$ & 0.82 \\
\hline CRP (mg/l) max & & $155.0 \pm 95.1$ & $102.4 \pm 97.4$ & 0.23 & $149.7 \pm 88.4$ & $198.2 \pm 145.0$ & 0.39 \\
\hline \multicolumn{2}{|l|}{ Duration of erythropenia in days } & $38.6 \pm 22.6$ & $28.7 \pm 22.4$ & 0.33 & $38.0 \pm 21.4$ & $40.0 \pm 31.5$ & 0.83 \\
\hline \multicolumn{2}{|l|}{ Duration of thrombopenia in days } & $31.7 \pm 19.4$ & $23.1 \pm 16.9$ & 0.28 & $29.6 \pm 17.7$ & $29.3 \pm 23.0$ & 0.97 \\
\hline \multicolumn{2}{|l|}{ Duration of leukopenia in days } & $26.8 \pm 18.9$ & $15.1 \pm 11.1$ & 0.09 & $23.5 \pm 17.4$ & $25.6 \pm 18.2$ & 0.59 \\
\hline \multicolumn{2}{|l|}{ Duration of neutropenia in days } & $15.9 \pm 16.7$ & $6.4 \pm 7.9$ & 0.11 & $13.6 \pm 15.4$ & $16.9 \pm 15.3$ & 0.49 \\
\hline \multicolumn{2}{|l|}{ Duration of fever in days } & $13.0 \pm 13.0$ & $9.3 \pm 9.7$ & 0.45 & $9.1 \pm 7.8$ & $25.8 \pm 22.5$ & 0.06 \\
\hline \multirow{2}{*}{ Positive microbiological findings } & Blood & $12 / 20(60 \%)$ & $2 / 5(40 \%)$ & 0.62 & $13 / 23(57 \%)$ & $5 / 6(83 \%)$ & 0.36 \\
\hline & Urine & $13 / 21(62 \%)$ & $0 / 3(0 \%)$ & 0.08 & $9 / 20(45 \%)$ & $6 / 7(86 \%)$ & 0.09 \\
\hline
\end{tabular}

CRP: C-reactive protein, LDH: lactate dehydrogenase, aPTT: activated Partial Thromboplastin Time, significant results are highlighted in bold $(p<0.05)$.

therapy. A decision analysis model concluded that 318 out of every 1000 patients suffer from an infectious complication, of which 68 infections would be caused by oral source [10]. The occurrence of bacteraemia caused by oral diseases in leukemic patients undergoing chemotherapy is controversially discussed $[11,23]$. On the one hand, periodontal diseases were found to be associated with an increased risk of infectious complications [21]. On the other hand, bacteraemia was not found to be associated with oral health in another study [11]. The current study was not able to find any evidence for associations between treatment need as well as periodontal inflammation and positive bacterial findings in blood or urine. Thereby, a real causative relationship appears difficult to assess.
The probably most important aspect seems to be the influence of oral health on the mortality of the patients. The above-mentioned analysis model assumes the possibility of additionally avoiding the death of 18 out of every 10000 patients by dental rehabilitation prior to chemotherapy [10]. This supports the recommendation of a dental clearance before therapy from the position paper of the joint task force of the Multinational Association of Supportive Care in Cancer/International Society of Oral Oncology (MASCC/ ISOO) and the European Society for Blood and Marrow Transplantation (EBMT) [5]. However, an increased risk during dental intervention caused by the immunosuppression and risk for bleeding must be considered before induction therapy as well [6]. The current pilot study was not 
TABLE 3: Initial blood parameters and therapeutic process depending on PISA.

\begin{tabular}{|c|c|c|c|c|}
\hline \multirow{2}{*}{ Parameter } & & \multicolumn{3}{|c|}{ PISA } \\
\hline & & $>98 \mathrm{~mm}^{2}(n=17)$ & $\leq 98 \mathrm{~mm}^{2}(n=17)$ & $p$ value \\
\hline \multicolumn{5}{|l|}{ Initial blood parameters } \\
\hline CRP $(\mathrm{mg} / \mathrm{l})$ & & $35.4 \pm 40.8$ & $38.9 \pm 36.6$ & 0.53 \\
\hline LDH (U/I) & & $610.3 \pm 789.3$ & $760.9 \pm 817.6$ & 0.18 \\
\hline aPTT (sec) & & $29.5 \pm 2.4$ & $27.6 \pm 7.5$ & 0.13 \\
\hline Hemoglobin (g/dl) & & $9.5 \pm 1.3$ & $10.5 \pm 2.0$ & 0.06 \\
\hline Hematocrit (\%) & & $27.8 \pm 3.6$ & $30.8 \pm 6.0$ & 0.09 \\
\hline \multicolumn{5}{|l|}{ Initial therapy parameters } \\
\hline \multirow{3}{*}{ Erythrocytes $\left(10^{3} / \mu \mathrm{l}\right)$} & Initial & $3.1 \pm 0.5$ & $3.5 \pm 0.8$ & 0.13 \\
\hline & 3 days & $3.0 \pm 0.5$ & $3.9 \pm 1.5$ & 0.01 \\
\hline & 7 days & $2.9 \pm 0.4$ & $3.4 \pm 1.0$ & 0.25 \\
\hline \multirow{3}{*}{ Thrombocytes $\left(10^{3} / \mu \mathrm{l}\right)$} & Initial & $73.4 \pm 76.3$ & $104.8 \pm 115.1$ & 0.72 \\
\hline & 3 days & $61.1 \pm 50.6$ & $101.6 \pm 111.7$ & 0.56 \\
\hline & 7 days & $42.0 \pm 34.7$ & $79.3 \pm 90.5$ & 0.31 \\
\hline \multirow{3}{*}{ Leukocytes $\left(10^{3} / \mu \mathrm{l}\right)$} & Initial & $13.2 \pm 28.7$ & $32.5 \pm 52.9$ & 0.34 \\
\hline & 3 days & $14.0 \pm 31.0$ & $22.6 \pm 46.2$ & 0.72 \\
\hline & 7 days & $8.5 \pm 18.6$ & $6.9 \pm 9.7$ & 0.31 \\
\hline CRP (mg/l) max & & $135.2 \pm 101.6$ & $153.0 \pm 93.2$ & 0.61 \\
\hline Duration of erythropenia in days & & $30.6 \pm 19.7$ & $43.1 \pm 24.5$ & 0.09 \\
\hline Duration of thrombopenia in days & & $28.6 \pm 19.2$ & $31.2 \pm 19.3$ & 0.70 \\
\hline Duration of leukopenia in days & & $26.6 \pm 20.7$ & $21.6 \pm 14.6$ & 0.65 \\
\hline Duration of neutropenia in days & & $16.3 \pm 18.3$ & $11.1 \pm 11.8$ & 0.47 \\
\hline Duration of fever in days & & $8.9 \pm 8.1$ & $15.4 \pm 15.1$ & 0.17 \\
\hline \multirow{2}{*}{ Positive microbiological findings } & Blood & $7 / 14(50 \%)$ & $7 / 11(64 \%)$ & 0.69 \\
\hline & Urine & $7 / 14(50 \%)$ & $6 / 10(60 \%)$ & 0.70 \\
\hline
\end{tabular}

CRP: C-reactive protein, LDH: lactate dehydrogenase, aPTT: activated Partial Thromboplastin Time, significant results are highlighted in bold $(p<0.05)$.

TABLE 4: Therapy associated complaints and mortality depending on treatment need and PISA.

\begin{tabular}{|c|c|c|c|c|c|c|c|c|c|c|}
\hline \multirow{2}{*}{ Parameter } & & \multirow{2}{*}{ Weakness } & \multirow{2}{*}{ Tachycardia } & \multirow{2}{*}{$\begin{array}{l}\text { Vertigo/ } \\
\text { nausea }\end{array}$} & \multirow{2}{*}{ Dyspnea } & \multicolumn{2}{|c|}{ Deceased } & \multicolumn{3}{|c|}{ Reason for death } \\
\hline & & & & & & Yes & No & Cancer & Infection & Others \\
\hline \multirow{3}{*}{ Dental treatment need } & Yes & $\begin{array}{l}14 / 19 \\
(48 \%)\end{array}$ & $9 / 29(31 \%)$ & $12 / 29(41 \%)$ & $\begin{array}{c}7 / 29 \\
(24 \%)\end{array}$ & $\begin{array}{c}5 / 29 \\
(17 \%)\end{array}$ & $\begin{array}{l}24 / 29 \\
(83 \%)\end{array}$ & 2 & 2 & 1 \\
\hline & No & $3 / 8(38 \%)$ & $1 / 8(13 \%)$ & $4 / 8(50 \%)$ & $1 / 8(13 \%)$ & $\begin{array}{c}1 / 10 \\
(10 \%)\end{array}$ & $\begin{array}{c}9 / 10 \\
(90 \%)\end{array}$ & 1 & 0 & 0 \\
\hline & $p$ value & 0.70 & 0.40 & 0.71 & 0.66 & \multicolumn{2}{|c|}{0.99} & & - & \\
\hline \multirow{3}{*}{$\begin{array}{l}\text { Periodontal treatment } \\
\text { need }\end{array}$} & Yes & $\begin{array}{l}13 / 25 \\
(52 \%)\end{array}$ & $6 / 25(24 \%)$ & $11 / 25(44 \%)$ & $\begin{array}{c}6 / 25 \\
(24 \%)\end{array}$ & $\begin{array}{l}5 / 25 \\
(20 \%)\end{array}$ & $\begin{array}{l}20 / 25 \\
(80 \%)\end{array}$ & 3 & 1 & 1 \\
\hline & No & $3 / 8(38 \%)$ & $3 / 8(38 \%)$ & $2 / 8(25 \%)$ & $1 / 8(13 \%)$ & $\begin{array}{c}1 / 9 \\
(11 \%)\end{array}$ & $8 / 9(89 \%)$ & 0 & 1 & 0 \\
\hline & $p$ value & 0.69 & 0.65 & 0.43 & 0.65 & \multicolumn{2}{|c|}{0.99} & & - & \\
\hline \multirow{3}{*}{ PISA } & $>98 \mathrm{~mm}^{2}$ & $7 / 17(41 \%)$ & $4 / 17(24 \%)$ & $7 / 17(41 \%)$ & $\begin{array}{c}5 / 17 \\
(29 \%)\end{array}$ & $\begin{array}{c}4 / 17 \\
(24 \%)\end{array}$ & $\begin{array}{l}13 / 17 \\
(76 \%)\end{array}$ & 2 & 1 & 1 \\
\hline & $\leq 98 \mathrm{~mm}^{2}$ & $9 / 16(56 \%)$ & $5 / 16(31 \%)$ & $6 / 16(38 \%)$ & $\begin{array}{c}2 / 16 \\
(13 \%)\end{array}$ & $\begin{array}{c}2 / 17 \\
(12 \%)\end{array}$ & $\begin{array}{l}15 / 17 \\
(88 \%)\end{array}$ & 1 & 1 & 0 \\
\hline & $p$ value & 0.49 & 0.71 & 0.99 & 0.40 & \multicolumn{2}{|c|}{0.66} & & - & \\
\hline
\end{tabular}

PISA: periodontal inflamed surface area.

able to detect a relationship between remaining treatment need and periodontal inflammation after dental clearance with mortality. This underlines the occurrence of fatal complications due to oral status being a very rare condition, which might only be detectable in a very large cohort. Thereby, a causative relationship is difficult to assess. Although the dental and periodontal treatment need after dental clearance were not associated with therapy outcome, its prevalence was high. Therefore, an individualized, patient oriented dental care concept under consideration of the immune status and blood coagulation of patients seems to be the most likely recommendable strategy [6]. Thereby, the implication of prevention-oriented concept after chemotherapy with the aim of preserving teeth, if possible, could be a promising and safe approach [25]. Within this patientoriented concept, the five core issues mentioned in the 
positions paper including prevention of infections, pain control, maintaining oral health, managing oral complications of treatment, and quality of life need to be addressed before, during, and after therapy [5].

Regardless of the missing association between oral conditions and outcome of induction therapy, dental rehabilitation and prevention remain a topic of high practical relevance. Early dental treatment and preventive special care should be applied within an interdisciplinary model. The integration of specialized dentists within oncologic centers might be a promising and helpful approach for the future to improve the dental care situation of patients with acute leukemia.

4.3. Strengths and Limitations. The current clinical study provides a comprehensive assessment of potential associations between dental/periodontal treatment need as well as periodontal inflammation with the initial treatment outcome and occurrence of complications of patients with acute leukemia, which previously underwent a dental clearance. Such data are limited in the literature but of high clinical importance. Nevertheless, there are several limitations to the current study. First, the small sample size is a major limitation. Especially in the subgroups, very small numbers of patients must be mentioned. Thus, the results of this pilot study must be seen as preliminary. Furthermore, design as a retrospective study limits the significance. However, the recruitment of patients with such severe disease is difficult due to their general disease burden. Thereby, based on the low prevalence of oral health related mortality mentioned in the literature [10] a very large cohort and a prospective design would be necessary to draw robust conclusions. Although a previous dental clearance was defined as an inclusion criterion, the content of the dental rehabilitation performed by the patient's dentist and the presence of remaining foci, e.g., by radiographs, was not checked. Accordingly, sufficient dental clearance can only be assumed but cannot be guaranteed. In general, the definition of treatment need as presence of carious lesions or probing depth $\geq 3.5 \mathrm{~mm}$ is very strict and might include patients with a very small risk of infections. Moreover, the cut-off for the analysis of PISA needs to be mentioned as a limitation. While the analysis was performed by median to ensure subgroups with a balanced sample size, a more clinical oriented categorization depending on the severity of periodontal diseases might provide other results. Thereby, the new classification of periodontal diseases, with a staging and grading matrix and the differentiation between localized and generalized or stable and unstable periodontal conditions, might be of interest, respectively [26]. However, this would also lead to a very small sample size in the subgroups.

\section{Conclusions}

Within the limitations of this pilot study, dental and periodontal treatment need, as well as periodontal inflammation, is not associated with the outcome of induction therapy in adult patients with acute leukemia. Accordingly, the rehabilitation of dental foci prior to chemotherapy appears sufficient to avoid systemic complications. Because of the high prevalence of remaining treatment need after dental clearance, an individualized and prevention-oriented dental treatment concept should be applied after chemotherapy.

\section{Data Availability}

No data were used to support this study.

\section{Conflicts of Interest}

The authors declare that there are no conflicts of interest regarding the publication of this paper.

\section{Authors' Contributions}

Gerhard Schmalz and Lulzim Tulani contributed equally as first authors.

\section{Acknowledgments}

The authors acknowledge support from the German Research Foundation (DFG) and Leipzig University within the program of Open Access Publishing.

\section{References}

[1] L. D. Cripe, "Adult acute leukemia," Current Problems in Cancer, vol. 21, no. 1, pp. 1-64, 1997.

[2] V. A. Upadhyay and A. T. Fathi, "Induction chemotherapy in acute myeloid leukaemia," Current Opinion in Hematology, vol. 25, no. 2, pp. 67-74, 2018.

[3] V. Mulanovich and D. P. Kontoyiannis, "Acute myeloid leukemia and the infectious diseases consultant," Leukemia \& Lymphoma, vol. 59, no. 6, pp. 1284-1291, 2018.

[4] J. A. Aas, B. J. Paster, L. N. Stokes, I. Olsen, and F. E. Dewhirst, "Defining the normal bacterial flora of the oral cavity," Journal of Clinical Microbiology, vol. 43, no. 11, pp. 5721-5732, 2005.

[5] S. Elad, J. E. Raber-Durlacher, M. T. Brennan et al., "Basic oral care for hematology-oncology patients and hematopoietic stem cell transplantation recipients: a position paper from the joint task force of the Multinational Association of Supportive Care in Cancer/International Society of Oral Oncology (MASCC/ISOO) and the European Society for Blood and Marrow Transplantation (EBMT)," Supportive Care in Cancer, vol. 23, no. 1, pp. 223-236, 2015.

[6] C. Zimmermann, M. I. Meurer, L. J Grando et al., "Dental treatment in patients with leukemia," Journal of Oncology, vol. 2015, Article ID 571739, 14 pages, 2015.

[7] N. M. Demian, J. W. Shum, I. L. Kessel, and A. Eid, "Oral surgery in patients undergoing chemoradiation therapy," Oral and Maxillofacial Surgery Clinics of North America, vol. 26, no. 2, pp. 193-207, 2014.

[8] S. Khaled, M. Al Malki, and G. Marcucci, "Acute myeloid leukemia: biologic, prognostic, and therapeutic insights," Oncology (Williston Park, N.Y.), vol. 30, no. 4, pp. 318-329, 2016.

[9] W. Stock, "Adolescents and young adults with acute lymphoblastic leukemia," Hematology, vol. 2010, no. 1, pp. 21-29, 2010.

[10] S. Elad, T. Thierer, M. Bitan, M. Y. Shapira, and C. Meyerowitz, "A decision analysis: the dental management 
of patients prior to hematology cytotoxic therapy or hematopoietic stem cell transplantation," Oral Oncology, vol. 44, no. 1, pp. 37-42, 2008.

[11] A. S. Sultan, Y. Zimering, G. Petruzziello et al., "Oral health status and risk of bacteremia following allogeneic hematopoietic cell transplantation," Oral Surgery, Oral Medicine, Oral Pathology and Oral Radiology, vol. 124, no. 3, pp. 253260, 2017.

[12] K. Dholam, S. Gurav, J. Dugad, and S. Banavli, "Correlation of oral health of children with acute leukemia during the induction phase," Indian Journal of Medical and Paediatric Oncology, vol. 35, no. 1, pp. 36-39, 2014.

[13] A. E. Gerritsen, P. F. Allen, D. J. Witter, E. M. Bronkhorst, and N. H. Creugers, "Tooth loss and oral health-related quality of life: a systematic review and meta-analysis," Health and Quality of Life Outcomes, vol. 8, no. 1, p. 126, 2010.

[14] R. Busjan, J. Hasenkamp, G. Schmalz, R. Haak, L. Trümper, and D. Ziebolz, "Oral health status in adult patients with newly diagnosed acute leukemia," Clinical Oral Investigations, vol. 22, no. 1, pp. 411-418, 2018.

[15] WHO, World Health Organization: Oral Health Surveys, Basic Methods, WHO; Oral Health Unit, Geneva, Switzerland, 4th edition, 1997.

[16] A. Diamanti-Kipioti, P. N. Papapanou, A. Moraitaki-Tsami, J. Lindhe, and F. Mitsis, "Comparative estimation of periodontal conditions by means of different index systems," Journal of Clinical Periodontology, vol. 20, no. 9, pp. 656-661, 1993.

[17] W. Nesse, F. Abbas, I. Van Der Ploeg, F. K. L. Spijkervet, P. U. Dijkstra, and A. Vissink, "Periodontal inflamed surface area: quantifying inflammatory burden," Journal of Clinical Periodontology, vol. 35, no. 8, pp. 668-673, 2008.

[18] M. A. Curtis, "Periodontal microbiology-the lid's off the box Again," Journal of Dental Research, vol. 93, no. 9, pp. 840-842, 2014.

[19] W. Zhang, C. G. Daly, D. Mitchell, and B. Curtis, "Incidence and magnitude of bacteraemia caused by flossing and by scaling and root planing," Journal of Clinical Periodontology, vol. 40, no. 1, pp. 41-52, 2013.

[20] I. Tomás, P. Diz, A. Tobías, C. Scully, and N. Donos, "Periodontal health status and bacteraemia from daily oral activities: systematic review/meta-analysis," Journal of Clinical Periodontology, vol. 39, no. 3, pp. 213-228, 2012.

[21] V. Allareddy, S. Prakasam, V. Allareddy et al., "Poor oral health linked with increased risk of infectious complications in adults with leukemia," Journal of the Massachusetts Dental Society, vol. 64, no. 64, pp. 38-42, 2015.

[22] F. Wong and J. A. Toljanic, "A survey of clinicians: prioritization of dental treatment in leukemia patients prior to chemotherapy," The International journal of prosthodontics, vol. 22, no. 3, pp. 303-306, 2009.

[23] P. D. M. Angst, D. A. M. Dutra, C. H. C. Moreira, and K. Z. Kantorski, "Periodontal status and its correlation with haematological parameters in patients with leukaemia," Journal of Clinical Periodontology, vol. 39, no. 11, pp. 10031010, 2012.

[24] B. G. Loos, "Systemic markers of inflammation in periodontitis," Journal of Periodontology, vol. 76, no. 11, pp. 2106-2115, 2005.

[25] K. Yamagata, K. Onizawa, T. Yanagawa, Y. Hasegawa, H. Kojima, and T. H. Nagasawa, "A prospective study to evaluate a new dental management protocol before hematopoietic stem cell transplantation," Bone Marrow Transplantation, vol. 38, no. 3, pp. 237-242, 2006.
[26] J. G. Yoshida, G. Armitage, T. Berglundh et al., "A new classification scheme for periodontal and peri-implant diseases and conditions - introduction and key changes from the 1999 classification," Journal of Periodontology, vol. 89, pp. S1-S8, 2018. 